\title{
EXTREME KHOVANOV SPECTRA
}

\author{
FEDERICO CANTERO MORÁN AND MARITHANIA SILVERO
}

\begin{abstract}
We prove that the spectrum constructed by González-Meneses, Manchón and the second author is stably homotopy equivalent to the Khovanov spectrum of Lipshitz and Sarkar at its extreme quantum grading.
\end{abstract}

\section{INTRODUCTION}

Khovanov homology is a powerful link invariant introduced by Mikhail Khovanov in Kho00 as a categorification of the Jones polynomial. More precisely, given an oriented diagram $D$ representing a link $L$, he constructed a finite $\mathbb{Z}$-graded family of chain complexes

$$
\ldots \longrightarrow C^{i, j}(D) \stackrel{d_{i}}{\longrightarrow} C^{i+1, j}(D) \stackrel{d_{i+1}}{\longrightarrow} C^{i+2, j}(D) \longrightarrow \ldots
$$

whose bigraded homology groups, $K h^{i, j}(D)$, are link invariants satisfying

$$
J(L)(q)=\sum_{i j} q^{j}(-1)^{i} \operatorname{rank}\left(K h^{i, j}(L)\right),
$$

where $J(L)$ is the Jones polynomial of $L$. The groups $K h^{i, j}(L)$ are known as Khovanov homology groups of $L$, and the indexes $i$ and $j$ as homological and quantum gradings, respectively.

A decade later, Lipshitz and Sarkar [LS14] constructed a $\mathbb{Z}$-graded family of spectra $\mathcal{X}^{j}(D)$ associated to a link diagram $D$, and they proved that

For each $j \in \mathbb{Z}$, the spectrum $\mathcal{X}^{j}(D)$ is a link invariant up to homotopy and there is a canonical isomorphism $H^{*}\left(\mathcal{X}^{j}(D)\right) \cong K h^{*, j}(D)$.

The construction of these spectra was later simplified in [LLS15] and [LLS17, where it was shown that each spectrum $\mathcal{X}^{j}$ can be obtained as the suspension spectrum of the realisation of a certain cubical functor on pointed topological spaces.

For a given link diagram $D$, the Khovanov chain complex is trivial for all but finitely many $j$ 's. Let $j_{\min }(D)$ be the minimal quantum grading such that the complex $\left\{C^{i, j}(D), d_{i}\right\}$ is non-trivial. In GMS17] González-Meneses, Manchón and Silvero introduced a simplicial complex $X_{D}$ satisfying the following

The simplicial complex $X_{D}$, if not contractible, is a link invariant up to stable homotopy and there is a canonical isomorphism $H^{*+n_{--1}}\left(X_{D}\right) \cong K h^{*, j_{\min }}(D)$, with $n_{-}$the number of negative crossings of $D$.

In this paper we show that, for the minimal quantum grading, both constructions are stably homotopy equivalent.

Both authors were supported by project MTM2016-76453-C2 (AEI/FEDER, UE) and acknowledge financial support from the Spanish Ministry of Economy and Competitiveness through the María de Maeztu Programme for Units of Excellence in R\&D (MDM-2014-0445). 

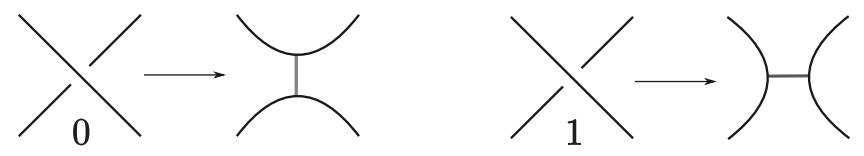

FIGURE 1. The smoothing of a crossing according to its 0 or 1 label.

\section{A stable homotopy equivalence}

2.1. States and enhancements. Let $\mathbf{2}^{\mathbf{n}}$ be the poset $\{1 \rightarrow 0\}^{n}$, which has an initial element $\overrightarrow{1}=(1,1, \ldots, 1)$ and a terminal element $\overrightarrow{0}=(0,0, \ldots, 0)$, and write $|v|=\sum_{i=1}^{n} v_{i}$.

Let $D$ be an oriented link diagram with $n$ ordered crossings, where $n_{+}\left(n_{-}\right)$of them are positive (negative). A state is an assignation of a label, 0 or 1 , to each crossing in $D$. There is a bijection between the set $\mathcal{S}$ of states of $D$ and the elements of $\mathbf{2}^{\mathbf{n}}$ by considering $v \in \mathbf{2}^{\mathbf{n}}$ as the state that assigns the $i^{t h}$ coordinate of $v$ to the $i^{\text {th }}$ crossing of $D$. Write $D(v)$ for the set of topological circles and chords obtained after smoothing each crossing of $D$ according to its label by following Figure 1 .

An enhacement of a state $v$ is a map $x$ assigning a sign \pm 1 to each of the $|D(v)|$ circles in $D(v)$. Write $\tau(v, x)=\sum x(c)$ where $c$ ranges over all circles in $D(v)$, and define, for the enhanced state $(v, x)$, the integers

$$
h(v, x)=h(v)=-n_{-}+|v|, \quad q(v, x)=n_{+}-2 n_{-}+|v|+\tau(v, x) .
$$

Let $j_{\text {min }}=\min \{q(v, x) \mid(v, x)$ is an enhanced state of $D\}$, and for any state $v$ write $v^{-}=\left(v, x_{-}\right)$with $x_{-}$the constant function with value -1 .

Proposition 1. GMS17, Proposition 4.1] In this setting, $j_{\min }=q\left(\overrightarrow{0}^{-}\right)$and $q(v, x)=$ $j_{\min }$ if and only if $(v, x) \in \mathcal{S}_{\min }$, where

$$
\mathcal{S}_{\text {min }}=\left\{\text { enhanced states }(v, x) \text { such that }|D(v)|=|D(\overrightarrow{0})|+|v| \text { and } x=x_{-}\right\} .
$$

In particular, $j_{\min }=n_{+}-2 n_{-}-|D(\overrightarrow{0})|$.

Proposition 2. Let $v \in \mathcal{S}_{\min }$. If $u<v$ then $u^{-} \in \mathcal{S}_{\min }$.

Proof. Note that the Khovanov differential $d$ either splits one circle into two or merges two circles into one. As $|D(v)|=|D(\overrightarrow{0})|+|v|$, necessarily $v$ is obtained from $\overrightarrow{0}$ by performing $|v|$ splittings in the crossings corresponding to non-zero coordinates of $v$. Hence, if $u$ and $v$ differ on $k$ coordinates, $v$ is obtained from $u$ by performing $k$ splittings, that is, $|D(u)|=|D(v)|-k=|D(\overrightarrow{0})|+|u|$.

2.2. The simplicial complex for extreme Khovanov homology. Let $D$ be an oriented link diagram. In GMS17, a simplicial complex $X_{D}$ was constructed, whose simplicial cochain complex is canonically isomorphic to the extreme Khovanov complex $\left\{C^{i, j_{\min }}(D), d_{i}\right\}$ shifted by $n_{-}-1$. Next, we review the construction of $X_{D}$ (cf. Figure 2).

The Lando graph $G_{D}$ associated to $D$ consists of a vertex for each chord in $D(\overrightarrow{0})$ having both endpoints in the same circle, and an edge between two vertices if the endpoints of the corresponding chords alternate in the same circle. The simplicial complex $X_{D}$ is defined as the independence complex of the graph $G_{D}$; in other words, the simplices of $X_{D}$ are the subsets of pairwise non-adjacent vertices of $G_{D}$. Alternatively, it is the clique complex of the complement graph of $G_{D}$. 
$(a)$

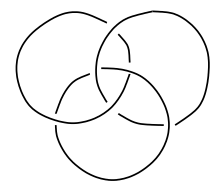

(b)

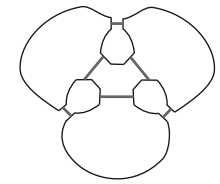

(c)

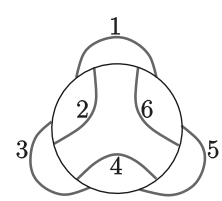

(d)

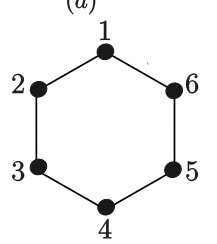

$(e)$

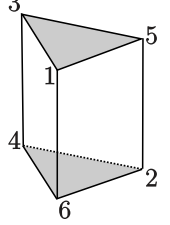

Figure 2. (a) A link diagram $D ;(b)$ and $(c)$ are two versions of $D(\overrightarrow{0})$; $(d)$ is the associated Lando graph $G_{D} ;(e)$ is the geometric realisation of the simplicial complex $X_{D}$.

2.3. Functors to the Burnside category. Let Top. be the category of pointed topological spaces with basepoint $*$. Let Set. be the category of pointed sets, which we wiew as a subcategory of Top. as the subcategory of discrete spaces. Let $\mathcal{B}$ be the Burnside 2-category for the trivial group, whose objects are finite sets, morphisms are spans and 2-morphisms are correspondences. We will freely refer to the results and notation of [LLS15] and [LLS17] in what follows (see also the survey [LS17]).

The category Set. sits inside $\mathcal{B}$ by sending a pointed set $A$ to $A \backslash\{*\}$, and a morphism $f: A \rightarrow B$ to the span

$$
A \backslash\{*\} \hookleftarrow A \backslash f^{-1}(*) \stackrel{f}{\longrightarrow} B \backslash\{*\} .
$$

Recall from [LLS15, Definition 5.1] that an $N$-dimensional spatial refinement of a functor $F: \mathbf{2}^{\mathbf{n}} \rightarrow \mathcal{B}$ is another functor $\tilde{F}: \mathbf{2}^{\mathbf{n}} \rightarrow$ Top. with values in wedges of spheres of dimension $N$ satisfying certain properties. The following observation is straightforward from that definition:

Lemma 3. A functor $F: \mathbf{2}^{\mathbf{n}} \rightarrow \mathcal{B}$ has a 0-dimensional spatial refinement $\tilde{F}$ if and only if $F$ factors as $\mathbf{2}^{\mathbf{n}} \rightarrow$ Set. $\hookrightarrow \mathcal{B}$. If this is the case, the refinement is $\tilde{F}: \mathbf{2}^{\mathbf{n}} \rightarrow$ Set. $\subset$ Top..

Let $\mathbf{2}_{+}^{\mathbf{n}}$ be the poset obtained as follows: Take a second copy of $\mathbf{2}^{\mathbf{n}}$, and rename its terminal object $\overrightarrow{0}$ as $\circ$. The poset $\mathbf{2}_{+}^{\mathbf{n}}$ is the union of both copies along the subposet $\mathbf{2}^{\mathbf{n}} \backslash\{\overrightarrow{0}\}$. Alternatively, it is the result of adding two cones to $\mathbf{2}^{\mathbf{n}} \backslash\{\overrightarrow{0}\}$ with apices $\overrightarrow{0}$ and $\circ$. If $\tilde{F}: \mathbf{2}^{\mathbf{n}} \rightarrow$ Top. is an $N$-dimensional spatial refinement, then its totalisation is defined as follows: extend $\tilde{F}$ to a functor $\tilde{F}_{+}: \mathbf{2}_{+}^{\text {n }} \rightarrow$ Top. by declaring $\tilde{F}_{+}(\circ)=*$ and define:

$$
\operatorname{Tot} \tilde{F}=\operatorname{hocolim} \tilde{F}_{+} \in \text { Top. }
$$

2.4. Khovanov spectra. Fix a link diagram $D$ and let $\mathcal{F}: \mathbf{2}^{\mathbf{n}} \rightarrow \mathcal{B}$ be the functor constructed in [LLS17, Proposition 6.1] whose value at a vertex $v$ is the set of all possible enhancements associated to the state $v$. Let $\mathcal{F}^{j}$ be the subfunctor whose values are those enhancements with quantum grading $j$. If $\tilde{\mathcal{F}}^{j}$ is an $N$-dimensional spatial refinement of $\mathcal{F}^{j}$, then the Khovanov spectrum of Lipshitz and Sarkar in quantum grading $j$ is [LLS15, Theorem 3]

$$
\mathcal{X}^{j} \simeq \Sigma^{-N-n_{-}} \Sigma^{\infty} \operatorname{Tot} \tilde{F}^{j} .
$$

When $j=j_{\min }$, we can restate Propositions 1 and 2 in the following way: 
Proposition 4. The value of $\mathcal{F}^{j_{\min }}$ at a vertex $v \in \mathbf{2}^{\mathbf{n}}$ is either the singleton $x_{-}$ for the case when $\left(v, x_{-}\right) \in \mathcal{S}_{\min }$, or empty otherwise. Moreover, the value of $\mathcal{F}^{j_{\min }}$ at an arrow $v>u$ is, depending on the values of $\mathcal{F}^{j_{\min }}(u)$ and $\mathcal{F}^{j_{\min }}(v)$,

\begin{tabular}{|c|c|c|}
\hline & $\mathcal{F}^{j_{\min }}(v)=\emptyset$ & $\mathcal{F}^{j_{\min }}(v)=x_{-}$ \\
\hline $\mathcal{F}^{j_{\min }}(u)=\emptyset$ & $\operatorname{Id}_{\emptyset}$ & $\nexists$ \\
\hline $\mathcal{F}^{j_{\min }}(u)=x_{-}$ & $\emptyset \rightarrow \mathcal{F}^{j_{\min }}(u)$ & $\mathcal{F}^{j_{\min }}(v) \cong \mathcal{F}^{j_{\min }}(u)$ \\
\hline
\end{tabular}

In particular, we obtain the following corollary:

Corollary 5. $\mathcal{F}^{j_{\min }}$ factors through Set. and therefore the factorisation $\tilde{\mathcal{F}}^{j_{\min }}$ is the 0-dimensional spatial refinement of $\mathcal{F}^{j_{\text {min }}}$. In fact, it further factors through the inclusion Set $\subset$ Set. sending a set $A$ to the pointed set $A \cup\{*\}$. If we write $\hat{\mathcal{F}}^{j_{\min }}$ for the latter factorisation, we get

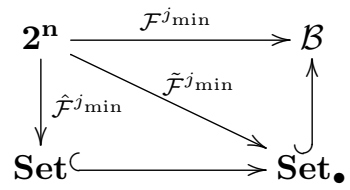

2.5. A homotopy equivalence. Let Poset be the category of posets, and let SComp be the category of simplicial complexes. There are functors

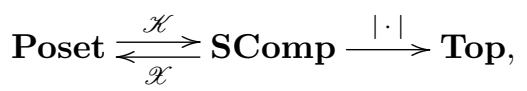

where $\mathscr{X}$ takes a simplicial complex to its poset of non-empty faces, $\mathscr{K}$ takes a poset $P$ to the simplicial complex whose 0 -simplices are the elements of $P$, and whose $i$-simplices are ascending chains of $i+1$ elements in $P$. The functor $|\cdot|$ takes a simplicial complex to its realisation. The composition $\mathscr{K} \circ \mathscr{X}$ takes a simplicial complex $Y$ to its barycentric subdivision $\operatorname{sd}(Y)$. We will denote the composition $|K(\cdot)|$ by $\|\cdot\|$. If $P$ is a poset and $F: P \rightarrow$ Top is a functor taking every element of $P$ to a singleton, then $\|P\|$ is a model for the homotopy colimit of $F$.

Let $\mathcal{S}_{\min }^{\prime} \subset \mathbf{2}^{\mathbf{n}}$ be the subposet of those states $v$ such that $\left(v, x_{-}\right) \in \mathcal{S}_{\min }$. The poset $\mathbf{2}^{\mathbf{n}}$ can be identified with the poset of faces of the $(n-1)$-dimensional simplex with the arrows reversed, where we identify $\overrightarrow{0}$ with the empty face and $\overrightarrow{1}$ with the top-dimensional face. Under this identification, the poset of faces of $X_{D}$ becomes precisely $\mathcal{S}_{\min }^{\prime}$ GMS17, Proposition 4.3]. Therefore, if $F: \mathcal{S}_{\min }^{\prime} \rightarrow$ Top is a functor with values on singletons, then

$$
\operatorname{hocolim} F \simeq\left\|\mathcal{S}_{\min }^{\prime} \backslash\{\overrightarrow{0}\}\right\|=\left|\operatorname{sd}\left(X_{D}\right)\right| \cong\left|X_{D}\right| .
$$

Theorem. There is a homotopy equivalence

$$
\mathcal{X}^{j_{\min }} \simeq \Sigma^{1-n_{-}} \Sigma^{\infty}\left|X_{D}\right|
$$

Proof. From (1) and Corollary 5 , we have that $\mathcal{X}^{j_{\min }} \simeq \Sigma^{-n_{-}} \Sigma^{\infty}$ hocolim $\tilde{\mathcal{F}}_{+}^{j_{\min }}$. We will prove that hocolim $\tilde{\mathcal{F}}_{+}^{j_{\min }} \simeq \Sigma\left\|\mathcal{S}_{\text {min }}^{\prime} \backslash\{\overrightarrow{0}\}\right\|$, and the result will follow from the homeomorphism $\left\|\mathcal{S}_{\min }^{\prime} \backslash\{\overrightarrow{0}\}\right\| \cong\left|X_{D}\right|$. 
As $\mathbf{2}_{+}^{\mathbf{n}}$ is constructed as the pushout of two cubes, there is a pushout diagram

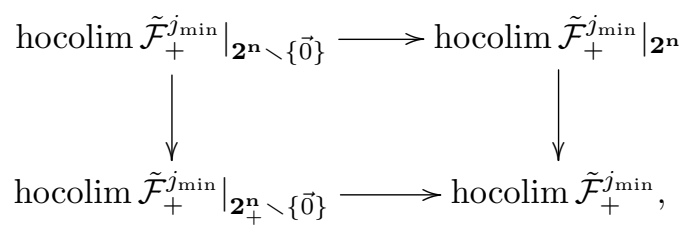

and as the two cubes have final elements $\overrightarrow{0}$ and $\circ$, we have

$$
\left.\operatorname{hocolim} \tilde{\mathcal{F}}_{+}^{j_{\min }}\right|_{2^{\mathrm{n}}} \simeq \tilde{\mathcal{F}}_{+}^{j_{\min }}(\overrightarrow{0})=\left\{x_{-}, *\right\},\left.\quad \operatorname{hocolim} \tilde{\mathcal{F}}_{+}^{j_{\min }}\right|_{\mathbf{2}_{+}^{\mathbf{n}} \backslash\{\overrightarrow{0}\}} \simeq \tilde{\mathcal{F}}_{+}^{j_{\min }}(\circ)=* .
$$

We now proceed to the computation of the upper left term in the diagram. Recall from the second part of Corollary 5 that $\tilde{\mathcal{F}}^{j_{\min }}$ factors as $\hat{\mathcal{F}}^{j_{\min }}: \mathbf{2}^{\mathbf{n}} \rightarrow$ Set $\subset$ Set. Since the inclusion Top $\subset$ Top. is a left adjoint, it preserves colimits, and therefore

$$
\text { hocolim }\left.\tilde{\mathcal{F}}_{+}^{j_{\min }}\right|_{\mathbf{2}^{\mathbf{n}} \backslash\{\overrightarrow{0}\}}=\left.\operatorname{hocolim} \hat{\mathcal{F}}^{j_{\min }}\right|_{\mathbf{2}^{\mathbf{n}} \backslash\{\overrightarrow{0}\}} \cup\{*\}
$$

Now, from Proposition 4 it follows that $\hat{\mathcal{F}}^{j_{\min }}(u)$ is either a point or empty depending on whether $u$ belongs to $\mathcal{S}_{\min }^{\prime}$ or not; therefore

$$
\text { hocolim }\left.\hat{\mathcal{F}}^{j_{\min }}\right|_{\mathbf{2}^{\mathbf{n}} \backslash\{\overrightarrow{0}\}}=\left.\operatorname{hocolim} \hat{\mathcal{F}}^{j_{\min }}\right|_{\mathcal{S}_{\min }^{\prime} \backslash\{\overrightarrow{0}\}}
$$

and since the latter functor is constant with values on singletons, (2) leads to

$$
\text { hocolim }\left.\hat{\mathcal{F}}^{j_{\min }}\right|_{\mathcal{S}_{\min }^{\prime} \backslash\{\overrightarrow{0}\}} \simeq\left\|\mathcal{S}_{\min }^{\prime} \backslash\{\overrightarrow{0}\}\right\| .
$$

Finally, we face again the original pushout diagram in Top.:

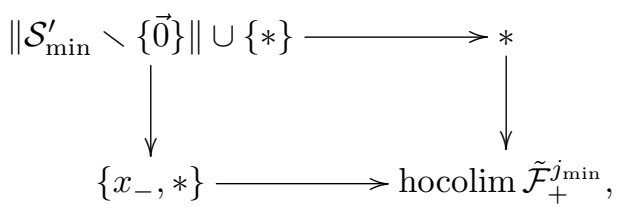

where the left vertical map collapses $\left\|\mathcal{S}_{\min }^{\prime} \backslash\{\overrightarrow{0}\}\right\|$ to $\left\{x_{-}\right\}$. Replacing $\left\{x_{-}, *\right\}$ by Cone $\left(\left\|\mathcal{S}_{\min }^{\prime} \backslash\{\overrightarrow{0}\}\right\|\right) \cup\{*\}$ and the $*$ in the upper right corner by Cone $\left(\left\|\mathcal{S}_{\min }^{\prime} \backslash\{\overrightarrow{0}\}\right\|\right)$ with basepoint the apex of the cone, we obtain a homotopy equivalent cofibrant pushout diagram, whose colimit is the (unreduced) suspension of $\left\|\mathcal{S}_{\min }^{\prime} \backslash\{\overrightarrow{0}\}\right\|$.

Remark 6. One can similarly define a maximal quantum grading $j_{\max }$ and define a simplicial complex $Y_{D}$ as the Alexander dual of $X_{D^{*}}$ where $D^{*}$ is the mirror image of $D$ (cf. [PS17, Theorem 7.4]). The fact that the Khovanov spectrum of a link diagram is the Spanier-Whitehead dual of the Khovanov spectrum of its mirror image, immediately implies that $\mathcal{X}^{j_{\max }} \simeq \Sigma^{n_{+}-1} \Sigma^{\infty} Y_{D}$.

\section{REFERENCES}

[GMS17] J. González-Meneses, P. M. G. Manchón, and M. Silvero, A geometric description of the extreme Khovanov cohomology, Proceedings of the Royal Society of Edinburgh: Section A Mathematics (2017), 117.

[Kho00] M. Khovanov, A categorification of the Jones polynomial, Duke Math. J. 101 (2000), no. $3,359-426$.

[LLS15] T. Lawson, R. Lipshitz, and S. Sarkar, Khovanov homotopy type, Burnside category, and products, arXiv:1505.00213, 2015. 
[LLS17] - The cube and the Burnside category, Categorification in geometry, topology, and physics, Contemp. Math., vol. 684, Amer. Math. Soc., Providence, RI, 2017, pp. 6385.

[LS14] R. Lipshitz and S. Sarkar, A Khovanov stable homotopy type, J. Amer. Math. Soc. 27 (2014), no. 4, 983-1042.

[LS17] , Spatial refinements and Khovanov homology, arXiv:1709.03602, 2017.

[PS17] J. H. Przytycki and M. Silvero, Homotopy type of circle graph complexes motivated by extreme Khovanov homology, J. Algebr. Comb. (2017). 